\title{
Impact of ethnicity on hepatitis C treatment outcomes
}

Asian or Hispanic ethnicity has a notable impact on treatment outcomes in patients with chronic hepatitis $\mathrm{C}$, according to the results of a recent study.

African American ethnicity is a wellknown negative predictor of treatment outcome for chronic hepatitis C; sustained virologic response (SVR) rates are significantly lower in African American patients compared with white patients, although the reasons for this are not fully understood. However, less is known about the relationship between Asian or Hispanic ethnicity and treatment outcomes as studies are often retrospective in nature or limited by small sample size.

Ke-Qin Hu et al. analyzed data from patients enrolled in the prospective, multicenter WIN-R trial to investigate the impact of ethnicity on treatment outcomes. Patients in this trial received PEG-IFN- $\alpha 2 b$ plus a fixed dose of ribavirin or a weight-based dose of ribavirin for 24 or 48 weeks. Patients with Asian $(n=118)$, Hispanic $(n=289)$ and white $(n=3,919)$ ethnicity were included in the subanalysis.

Overall, SVR rates were significantly higher among Asian and white patients than among Hispanic patients, and SVR rates were higher among Asian patients than among white patients. Psychiatric adverse events were less common and anemia was more common in Asian patients than in white or Hispanic patients.

The authors conclude that ethnicity is an important factor to consider in personalized treatment strategies for chronic hepatitis C.

Isobel Franks

Original article Hu, K.-Q. et al. Impact of Hispanic or Asian ethnicity on the treatment outcomes of chronic hepatitis C: results from the WIN-R trial. J. Clin. Gastroenterol. doi:10.1097/MCG.0b013e31820d35e3 